\title{
Diagnostic ability of macular ganglion cell asymmetry in Preperimetric Glaucoma
}

Mei-Ju Chen ${ }^{1,2}$, Hsin-Yu Yang ${ }^{1}$, Yu-Fan Chang ${ }^{1,3}$, Chih-Chien Hsu ${ }^{1,3}$, Yu-Chieh Ko ${ }^{1,2,3}$ and

Catherine Jui-Ling Liu ${ }^{1,2^{*}}$ (D)

\begin{abstract}
Background: To evaluate the diagnostic ability of macular ganglion cell asymmetry to diagnose preperimetric glaucoma (PPG), using Cirrus spectral domain optical coherence tomography (OCT).

Methods: This prospective study included 67 eyes of 67 patients with PPG and 67 eyes of 67 age- and refractive error-matched controls. We measured circumpapillary RNFL (cpRNFL) thickness, macular ganglion cell-inner plexiform layer (GCIPL) thickness and optic nerve head (ONH) parameters using OCT. Macular ganglion cell asymmetries were expressed as absolute difference and ratios between inferior hemisphere and superior hemisphere, inferotemporal (IT) and superotemporal (ST), IT and superonasal (SN), IT and inferonasal (IN), ST and IN as well as temporal and nasal. An asymmetry index was assigned by taking the absolute value of $\log _{10}$ of the ratio. The area under the receiver operating characteristics curve (AUROC), partial AUROC (PAUROC) $\geq$ specificities 90 and 95\%, cutoff values and sensitivities at specificities 90 and $95 \%$ was analyzed.
\end{abstract}

Results: Parameters with largest AUROCs were IT GCIPL thickness (0.784), average RNFL thickness (0.767), and average C/D (0.746). For macular asymmetry parameters, log IT/SN index had the largest AUROC (0.734), followed by log IT/IN index (0.725), and absolute difference of IT-SN GCIPL thickness (0.715). Performance was comparable between the best measures of asymmetry analysis (log IT/SN index) and those of cpRNFL, GCIPL, and ONH parameters (all $P>0.05$ ). The IT/SN asymmetry index not only had the largest pAUROC based on the pAUROCs $\geq 90$ and 95\% specificity (0.044 and 0 . 019) but also had the highest diagnostic sensitivity at 90 and 95\% specificities (52.2 and 46.3\%).

Conclusions: GCIPL asymmetry measurements have diagnostic ability comparable to cpRNFL, GCIPL, and ONH analysis for PPG. The best macular ganglion cell asymmetry parameter was IT/SN asymmetry index, which could be a new parameter to detect early structural changes in PPG.

Keywords: Macular ganglion cell asymmetry, Optical coherence tomography, Preperimetric glaucoma

\section{Background}

Glaucoma is characterized by the progressive death of retinal ganglion cells (RGCs) and loss of their axons, with associated visual field (VF) defects. Previous studies indicate that VF defect may not be clinically detectable until 25 to $35 \%$ of all RGCs are lost [1-4]. Early detection of structural changes associated with RGCs loss is especially important for preperimetric glaucoma (PPG), which presents with glaucomatous optic disc, retinal nerve fiber layer (RNFL) abnormalities, and normal VF. The introduction

\footnotetext{
* Correspondence: jlliu@vghtpe.gov.tw

1Department of Ophthalmology, Taipei Veterans General Hospital, 201, Section 2, Shih-Pai Road, Taipei 11217, Taiwan

${ }^{2}$ School of Medicine, National Yang-Ming University, Taipei, Taiwan Full list of author information is available at the end of the article
}

of spectral domain optical coherence tomography (OCT) allows for the reproducible and successful segmentation of the inner macular layers. Several studies have shown thinning of the inner retina or RGC complex within the macular area in early glaucoma [5, 6] and PPG [7]. The thickness of macular ganglion cell-inner plexiform layer (GCIPL) may serve as an early indicator of glaucomatous structural damage [8]. OCT has revealed a step-like arcuate defect in the temporal macular GCIPL map in early glaucomatous eyes [9], due to asymmetry in GCIPL thickness distribution between the superior and inferior hemispheres. Using Spectralis posterior pole asymmetry analysis [10-13], Cirrus GCIPL asymmetry [14] or customized software $[15,16]$, asymmetric glaucomatous 

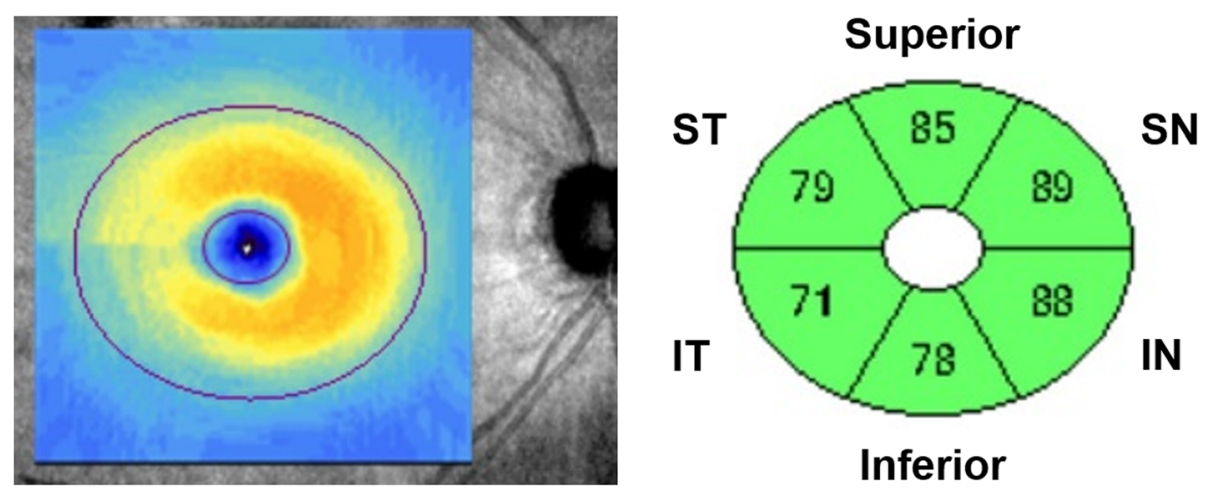

Fig. 1 The Cirrus HD-OCT macular cube $200 \times 200$ protocol provides regional GCIPL thickness in six wedge-shaped sectors

macular damage between the inferior and superior hemispheres has been reported in early glaucoma. However, glaucoma frequently starts as a localized thinning of RGC, which might be undetected by averaging values of GCIPL thickness in the designated hemispheric area and its counterpart. Comparison of GCIPL thickness between each parafoveal sector may be necessary to avoid this problem and accurately evaluate ganglion cell asymmetry. In this study, we evaluate the diagnostic ability of the absolute difference, asymmetry ratio, and asymmetry index between six parafoveal macular GCIPL thickness measurements in PPG patients, and compare the asymmetry analysis of GCIPL thickness with traditional cpRNFL, GCIPL, and optic nerve head $(\mathrm{ONH})$ parameters in PPG patients.

\section{Methods}

Patients with PPG who visited the outpatient clinic of Taipei Veterans General Hospital between June 2014 and December 2015 were recruited for this study. We also enrolled age- and refractive error- matched control subjects by recruiting healthy volunteers from the same hospital. The study protocol was approved by the Institutional Review Board of our hospital and was designed in accordance with the Declaration of Helsinki. Written informed consent was obtained from all subjects.

Eyes with focal or diffuse RNFL defects corresponding to glaucomatous optic disc changes and a normal VF test were assigned to the PPG group. Glaucomatous optic disc changes were defined as $>0.7$ vertical cup to disc ratio $(C / D),>0.2$ asymmetric $C / D$ between the glaucomatous and normal eyes, and neuroretinal rim thinning, notching, or excavation on optic disc photography. Focal or diffuse RNFL defects were based on red-free fundus images. All images including optic nerve head appearance and RNFL defects were evaluated by three glaucoma specialist (M.J.C, Y.C.K and C.J.L.), who were masked to the results of the subjects' clinical evaluations. A normal VF was defined as a mean deviation
(MD) and pattern standard deviation (PSD) within the 95\% confidence limit, and a glaucoma hemifield test result within the normal limits by reliable VF test [17]. A reliable VF test was defined as having a fixation loss rate of $<20 \%$, false positive rate of $<33 \%$, and false negative rate of $<33 \%$.

All subjects underwent a comprehensive ophthalmic examination, including assessment of best corrected visual acuity, automated refraction and keratometry, Goldman applanation tonometry, slit-lamp examination, gonioscopy, dilated fundus exam, red-free fundus photography, and automated VF examination (Humphrey 24-2 SITA standard algorithmn). Axial length (AL) was measured using an IOLMaster (Carl Zeiss Meditec, Dublin, California, USA), and central corneal thickness (CCT) was determined using a DGH 55 Pachmate (DGH Technology, Exton, Pennsylvania, USA). Subjects had to meet the following criteria to be enrolled in this study: age $\geq 20$ years, best corrected visual acuity $\geq 20 / 40$, open angle structure upon gonioscopic examination, and astigmatism $\leq 3$ diopters (D). Control subjects were required to have a normal anterior segment on slit-lamp

Table 1 Demographic and clinical characteristics of the study population

\begin{tabular}{llll}
\hline & Normal $(n=67)$ & PPG $(n=67)$ & $P$ value \\
\hline Years of Age & $45.3 \pm 15.4$ & $48.3 \pm 11.1$ & 0.191 \\
Male/Female & $29 / 39$ & $33 / 35$ & 0.082 \\
SE (D) & $-4.25 \pm 4.13$ & $-4.62 \pm 3.40$ & 0.572 \\
AL $(\mathrm{mm})$ & $25.35 \pm 1.76$ & $25.60 \pm 1.37$ & 0.375 \\
IOP $(\mathrm{mmHg})$ & $16.2 \pm 3.4$ & $18.4 \pm 3.1$ & 0.030 \\
CCT $(\mu \mathrm{m})$ & $557 \pm 37$ & $566 \pm 36$ & 0.218 \\
MD $(\mathrm{dB})$ & $-1.06 \pm 1.41$ & $-1.35 \pm 1.61$ & 0.265 \\
PSD $(\mathrm{dB})$ & $1.83 \pm 0.74$ & $1.77 \pm 0.58$ & 0.596 \\
VFI (\%) & $98.9 \pm 0.97$ & $98.6 \pm 1.61$ & 0.189 \\
\hline PPG & Prepermetri glucoma; SE,
\end{tabular}

$P P G$, preperimetric glaucoma; $S E$, spherical equivalent; $A L$, axial length; $I O P$, intraocular pressure; $C C T$, central corneal thickness; $M D$, mean deviation; $P S D$, pattern standard deviation; $V F I$, visual field index 
Table 2 Comparison of cpRNFL thickness, GCIPL thickness and asymmetry measurements, and $\mathrm{ONH}$ parameters between two groups

\begin{tabular}{|c|c|c|c|}
\hline Parametres & $\begin{array}{l}\text { Normal } \\
(n=67)\end{array}$ & $\begin{array}{l}\text { PPG } \\
(n=67)\end{array}$ & $P$ value \\
\hline \multicolumn{4}{|l|}{ cpRNFL Thickness } \\
\hline Average & $96.0 \pm 9.2$ & $86.8 \pm 8.7$ & $<0.001$ \\
\hline Superior & $115.1 \pm 16.4$ & $104.2 \pm 15.3$ & $<0.001$ \\
\hline Nasal & $65.1 \pm 10.2$ & $64.4 \pm 12.2$ & 0.712 \\
\hline Inferior & $119.8 \pm 17.1$ & $105.9 \pm 16.1$ & $<0.001$ \\
\hline Temporal & $83.0 \pm 14.6$ & $73.7 \pm 12.7$ & $<0.001$ \\
\hline \multicolumn{4}{|l|}{ GCIPL Thickness } \\
\hline Average & $80.6 \pm 5.9$ & $76.2 \pm 6.6$ & $<0.001$ \\
\hline Minimum & $77.9 \pm 6.7$ & $71.8 \pm 9.0$ & $<0.001$ \\
\hline Superonasal & $82.8 \pm 6.7$ & $80.0 \pm 9.0$ & 0.043 \\
\hline Superior & $81.1 \pm 6.6$ & $77.4 \pm 7.8$ & 0.004 \\
\hline Superotemporal & $80.1 \pm 5.4$ & $75.5 \pm 7.2$ & $<0.001$ \\
\hline Inferotemporal & $80.8 \pm 5.6$ & $73.5 \pm 7.5$ & $<0.001$ \\
\hline Inferior & $77.8 \pm 6.6$ & $72.7 \pm 7.4$ & $<0.001$ \\
\hline Inferonasal & $80.9 \pm 7.0$ & $78.0 \pm 7.8$ & 0.024 \\
\hline \multicolumn{4}{|c|}{ GCIPL Absolute Difference } \\
\hline $1-S$ & $6.1 \pm 4.2$ & $12.3 \pm 13.6$ & 0.001 \\
\hline IT-ST & $2.5 \pm 1.7$ & $5.2 \pm 5.5$ & $<0.001$ \\
\hline $\mathrm{T}-\mathrm{N}$ & $6.9 \pm 4.5$ & $11.9 \pm 8.9$ & $<0.001$ \\
\hline IT-SN & $3.7 \pm 2.4$ & $8.2 \pm 7.2$ & $<0.001$ \\
\hline IT-IN & $3.3 \pm 2.8$ & $6.5 \pm 5.6$ & $<0.001$ \\
\hline ST-IN & $3.7 \pm 3.4$ & $5.0 \pm 4.4$ & 0.048 \\
\hline \multicolumn{4}{|c|}{ GCIPL Asymmetry Ratio } \\
\hline $1 / S$ & $0.98 \pm 0.03$ & $0.97 \pm 0.08$ & 0.109 \\
\hline IT/ST & $1.01 \pm 0.04$ & $0.98 \pm 0.10$ & 0.019 \\
\hline $\mathrm{T} / \mathrm{N}$ & $0.98 \pm 0.05$ & $0.95 \pm 0.08$ & 0.002 \\
\hline IT/SN & $0.98 \pm 0.05$ & $0.93 \pm 0.11$ & 0.001 \\
\hline IT/IN & $1.00 \pm 0.06$ & $0.95 \pm 0.09$ & $<0.001$ \\
\hline ST/IN & $0.98 \pm 0.07$ & $0.97 \pm 0.09$ & 0.118 \\
\hline \multicolumn{4}{|c|}{ GCIPL Asymmetry Index } \\
\hline $\log _{10}(\mathrm{I} / \mathrm{S})$ & $0.01 \pm 0.01$ & $0.02 \pm 0.03$ & 0.001 \\
\hline $\log _{10}(\mathrm{IT} / \mathrm{ST})$ & $0.01 \pm 0.01$ & $0.03 \pm 0.03$ & $<0.001$ \\
\hline $\log _{10}(\mathrm{~T} / \mathrm{N})$ & $0.02 \pm 0.01$ & $0.03 \pm 0.03$ & $<0.001$ \\
\hline $\log _{10}(\mathrm{IT} / \mathrm{SN})$ & $0.02 \pm 0.01$ & $0.05 \pm 0.04$ & $<0.001$ \\
\hline $\log _{10}(\mathrm{IT} / \mathrm{IN})$ & $0.00 \pm 0.02$ & $0.04 \pm 0.03$ & $<0.001$ \\
\hline $\log _{10}(\mathrm{ST} / \mathrm{IN})$ & $0.02 \pm 0.02$ & $0.03 \pm 0.03$ & 0.026 \\
\hline \multicolumn{4}{|l|}{$\mathrm{ONH}$} \\
\hline Rim Area & $1.21 \pm 0.23$ & $1.05 \pm 0.19$ & $<0.001$ \\
\hline Disc Area & $1.78 \pm 0.50$ & $1.98 \pm 0.50$ & $<0.001$ \\
\hline Average C/D & $0.50 \pm 0.20$ & $0.63 \pm 0.18$ & $<0.001$ \\
\hline
\end{tabular}

Table 2 Comparison of cpRNFL thickness, GCIPL thickness and asymmetry measurements, and $\mathrm{ONH}$ parameters between two groups (Continued)

\begin{tabular}{ccll}
\hline Parametres & $\begin{array}{l}\text { Normal } \\
(n=67)\end{array}$ & $\begin{array}{l}\text { PPG } \\
(n=67)\end{array}$ & $P$ value \\
\hline Vertical C/D & $0.47 \pm 0.20$ & $0.60 \pm 0.18$ & $<0.001$ \\
Cup Volume & $0.18 \pm 0.15$ & $0.36 \pm 0.26$ & $<0.001$
\end{tabular}

PPG, preperimetric glaucoma; GCIPL, ganglion cell-inner plexiform layer; $C p R N F L$, circumferential peripapillary retinal nerve fiber layer; $O N H$, optic nerve head; $C / D$, cup-to-disc ratio; $I$, inferior hemisphere; $S$, superior hemisphere; $I T$, inferotemporal; $S T$, superotemporal; $T$, temporal; $N$, nasal; $S N$, superonasal; IN inferonasal

examination, no glaucomatous changes of the $\mathrm{ONH}$ and normal VF. Eyes were excluded if they showed retinal or neurologic diseases; ocular inflammation; prior ocular surgery within 3 months; prior refractive surgery, or concurrent disease that could interfere with IOP measurement or OCT imaging or cause VF defects.

Cirrus HD-OCT (Carl Zeiss Meditec, Dublin, California, USA) was performed following pupillary dilation. The Cirrus HD-OCT Optic Disc Cube $200 \times 200$ protocol was used to measure $\mathrm{ONH}$ rim area, disc area, average $\mathrm{C} / \mathrm{D}$, vertical $\mathrm{C} / \mathrm{D}$, cup volume, average circumpapillary RNFL (cpRNFL) thickness, and cpRNFL thickness in quadrants and in 12 clock-hour sectors. The Macular Cube $200 \times$ 200 protocol was used to calculate average, minimum, and regional GCIPL thickness in six wedge-shaped sectors (Fig. 1). GCIPL thickness in superior hemisphere (S) was calculated as sum of superonasal (SN), superior and superotemporal (ST). GCIPL thickness in inferior hemisphere (I) was calculated as sum of inferonasal (IN), inferior and inferotemporal (IT). We attempted to compare the difference between I and S, IT and ST, IT and SN, IT and IN, ST and IN as well as temporal (T, sum of ST and IT) vs. nasal (N, sum of SN and IN). In addition to hemispheric comparison, sectorial comparison between six parafoveal sectors were also made for asymmetry evaluation of the GCIPL thickness. Macular ganglion cell asymmetry was expressed as absolute difference (i.e., I-S) and ratio (i.e., I/ S) of the thickness between the designated areas. Finally, an asymmetry index was calculated as absolute value of $\log _{10}$ of the ratio (i.e., $\log _{10} I / S$ ). Images were excluded if they exhibited signal strength $<7$, motion artifact, poor centration, segmentation error, artifacts caused by ocular pathology, or missing data on the peripapillary region. There was a time interval of $<3$ months between HD-OCT and other ophthalmic examination (i.e., VF).

For each subjects, one eye was randomly chosen if both eyes were eligible. The original data was provided as Additional files 1 and 2. Statistical analyses were performed using SPSS version 12.0 (SPSS, Inc., Chicago, IL, USA) and STATA version 12.1 (Stata Corp, College Station, TX, USA). For continuous variables, the normality of data distribution was verified using the Shapiro-Wilk 
Table 3 AUROC and pAUROC values for cpRNFL thickness, GCIPL thickness, GCIPL asymmetry measurements, and ONH parameters

\begin{tabular}{|c|c|c|c|}
\hline Parameters & AUROC (95\% Cl) & pAUROC at $90 \%$ specificity $(95 \% \mathrm{Cl})$ & pAUROC at $95 \%$ specificity $(95 \% \mathrm{Cl})$ \\
\hline \multicolumn{4}{|l|}{ cpRNFL Thickness } \\
\hline Average & $0.767(0.685-0.850)$ & $0.019(0.004-0.035)$ & $0.007(0.000-0.013)$ \\
\hline Superior & $0.697(0.605-0.789)$ & $0.013(0.000-0.027)$ & $0.002(-0.004-0.008)$ \\
\hline Nasal & $0.523(0.421-0.622)$ & $0.007(-0.004-0.014)$ & $0.001(-0.001-0.005)$ \\
\hline Inferior & $0.727(0.639-0.816)$ & $0.018(0.002-0.034)$ & $0.005(-0.003-0.013)$ \\
\hline Temporal & $0.676(0.581-0.770)$ & $0.012(0.001-0.023)$ & $0.005(0.000-0.009)$ \\
\hline \multicolumn{4}{|l|}{ GCIPL Thickness } \\
\hline Average & $0.693(0.604-0.783)$ & $0.014(0.004-0.023)$ & $0.005(0.000-0.009)$ \\
\hline Minimum & $0.720(0.634-0.807)$ & $0.021(0.007-0.035)$ & $0.006(0.000-0.013)$ \\
\hline Superonasal & $0.632(0.537-0.727)$ & $0.010(0.001-0.017)$ & $0.004(0.001-0.007)$ \\
\hline Superior & $0.632(0.537-0.727)$ & $0.010(0.002-0.018)$ & $0.003(0.000-0.007)$ \\
\hline Superotemporal & $0.699(0.610-0.787)$ & $0.016(0.003-0.028)$ & $0.006(0.001-0.011)$ \\
\hline Inferotemporal & $0.784(0.707-0.861)$ & $0.035(0.020-0.050)$ & $0.014(0.007-0.021)$ \\
\hline Inferior & $0.695(0.606-0.784)$ & $0.020(0.010-0.030)$ & $0.010(0.005-0.014)$ \\
\hline Inferonasal & $0.614(0.519-0.710)$ & $0.007(-0.001-0.015)$ & $0.002(-0.001-0.005)$ \\
\hline \multicolumn{4}{|c|}{ GCIPL Absolute difference } \\
\hline I-S & $0.628(0.532-0.724)$ & $0.023(0.013-0.034)$ & $0.011(0.005-0.016)$ \\
\hline IT-ST & $0.624(0.527-0.722)$ & $0.031(0.019-0.042)$ & $0.014(0.008-0.020)$ \\
\hline $\mathrm{T}-\mathrm{N}$ & $0.661(0.561-0.754)$ & $0.024(0.012-0.026)$ & $0.010(0.004-0.016)$ \\
\hline IT-SN & $0.715(0.626-0.803)$ & $0.034(0.021-0.047)$ & $0.015(0.009-0.022)$ \\
\hline IT-IN & $0.704(0.617-0.790)$ & $0.023(0.012-0.033)$ & $0.010(0.004-0.015)$ \\
\hline ST-IN & $0.600(0.503-0.697)$ & $0.009(0.002-0.016)$ & $0.004(0.000-0.008)$ \\
\hline \multicolumn{4}{|c|}{ GCIPL Asymmetry Ratio } \\
\hline I/S & $0.604(0.507-0.701)$ & $0.025(0.013-0.036)$ & $0.010(0.005-0.016)$ \\
\hline IT/ST & $0.606(0.508-0.703)$ & $0.028(0.017-0.040)$ & $0.013(0.007-0.018)$ \\
\hline $\mathrm{T} / \mathrm{N}$ & $0.684(0.592-0.776)$ & $0.033(0.018-0.047)$ & $0.014(0.006-0.022)$ \\
\hline IT/SN & $0.690(0.598-0.782)$ & $0.039(0.026-0.052)$ & $0.017(0.010-0.024)$ \\
\hline IT/IN & $0.694(0.603-0.785)$ & $0.031(0.018-0.043)$ & $0.013(0.007-0.019)$ \\
\hline $\mathrm{ST} / \mathrm{IN}$ & $0.610(0.513-0.708)$ & $0.013(0.000-0.026)$ & $0.035(-0.001-0.009)$ \\
\hline \multicolumn{4}{|c|}{ GCIPL Asymmetry Index } \\
\hline $\log _{10}(I / S)$ & $0.636(0.540-0.731)$ & $0.024(0.012-0.036)$ & $0.010(0.004-0.016)$ \\
\hline $\log _{10}(\mathrm{IT} / \mathrm{ST})$ & $0.651(0.556-0.747)$ & $0.036(0.024-0.048)$ & $0.017(0.011-0.023)$ \\
\hline $\log _{10}(T / N)$ & $0.678(0.585-0.770)$ & $0.026(0.012-0.041)$ & $0.010(0.004-0.016)$ \\
\hline $\log _{10}(\mathrm{IT} / \mathrm{SN})$ & $0.734(0.648-0.820)$ & $0.044(0.031-0.057)$ & $0.019(0.012-0.026)$ \\
\hline $\log _{10}(\mathrm{IT} / \mathrm{IN})$ & $0.725(0.641-0.809)$ & $0.025(0.013-0.038)$ & $0.011(0.005-0.017)$ \\
\hline $\log _{10}(\mathrm{ST} / \mathrm{IN})$ & $0.624(0.529-0.720)$ & $0.010(0.001-0.019)$ & $0.004(0.000-0.008)$ \\
\hline \multicolumn{4}{|l|}{$\mathrm{ONH}$} \\
\hline Rim Area & $0.716(0.626-0.806)$ & $0.074(0.004-0.030)$ & $0.006(0.000-0.011)$ \\
\hline Disc Area & $0.636(0.538-0.734)$ & $0.005(-0.004-0.014)$ & $0.000(-0.004-0.004)$ \\
\hline Average C/D & $0.746(0.662-0.831)$ & $0.029(0.013-0.045)$ & $0.012(0.004-0.019)$ \\
\hline Vertical C/D & $0.742(0.656-0.828)$ & $0.028(0.015-0.041)$ & $0.017(0.009-0.025)$ \\
\hline Cup Volume & $0.729(0.641-0.816)$ & $0.029(0.009-0.050)$ & $0.007(-0.004-0.018)$ \\
\hline
\end{tabular}

$A U R O C$, area under the receiver operating characteristic curves; $P A U R O C$, partial area under the receiver operating characteristic curves; confidence interval; GCIPL, ganglion cell-inner plexiform layer; $c p R N F L$, circumferential peripapillary retinal nerve fiber layer; $O N H$, optic nerve head; $C l$, confidence interval; $C / D$, cup-to-disc ratio; I, inferior hemisphere; $S$, superior hemisphere; $I T$, inferotemporal; $S T$, superotemporal; $T$, temporal; $N$, nasal; $S N$, superonasal; $I N$, inferonasal 
test. We employed Student's $t$ test for normally distributed data, and the Mann-Whitney $U$ test for non-normally distributed data to analyze differences between PPG and normal groups. The chi-square test was used to compare the sex ratio. To evaluate the ability of each parameter to discriminate between PPG and normal eyes, we calculated the area under the receiver operating characteristic curve (AUROC), partial AUROC (pAUROC) $\geq$ specificities 90 and $95 \%$, cutoff values and sensitivities at specificities 90 and $95 \%$ for each parameter. The diagnostic performance quantified by AUROC and pAUROC values was compared by using the methods from DeLong et al. [18]. The chi-square test was used to compare the sensitivities at fixed specificities of OCT parameters. For the cpRNFL thickness, GCIPL thickness, GCIPL asymmetry measurements, and $\mathrm{ONH}$ parameters comparisons, Bonferroni adjustments were made based on the number of comparisons to correct type I error. For other analyses, $P$ value of $<0.05$ was considered statistically significant.

\section{Results}

This study included 67 PPG eyes of 67 patients and 67 eyes of 67 age- and refractive error-matched normal controls. The demographic and clinical characteristics of the subjects are summarized in Table 1 . There were no significant between-group differences in age, sex, spherical equivalence, AL, CCT, MD, PSD, or visual field index (VFI). However, PPG eyes showed significantly higher intraocular pressure (IOP) compared to controls.

After the Bonferroni adjustment $(\alpha=0.0014 ; 36$ comparisons), all GCIPL thickness and most cpRNFL thickness (except at the nasal quadrant) measured by HD-OCT were significantly lower in PPG eyes compared to normal eyes $(P<0.001)$ (Table 2$)$. PPG eyes had significantly larger disc area, average $C / D$, vertical $C / D$, and cup volume as well as significantly smaller rim area than controls. PPG eyes also had significantly greater GCIPL absolute difference than controls. Significant differences in GCIPL asymmetry ratio were found between the PPG and normal eyes, with the exception of I/S. GCIPL asymmetry index values were significantly different between PPG and normal eyes. Table 3 shows the AUROC and pAUROC values for all the thickness and asymmetry parameters. The IT GCIPL thickness had the largest AUROC value (0.784), followed by average RNFL thickness (0.767), average C/D (0.746), vertical C/D (0.742), and IT/SN asymmetry index (0.734) (Fig. 2). The macular ganglion cell asymmetry parameters with the largest AUROCs were IT/SN asymmetry index (0.734), IT/IN asymmetry index (0.725), and IT-SN GCIPL thickness (0.715). The best individual asymmetry parameters were IT-SN for absolute difference, IT/IN (0.694) for asymmetry ratio, and IT/SN asymmetry index for asymmetry

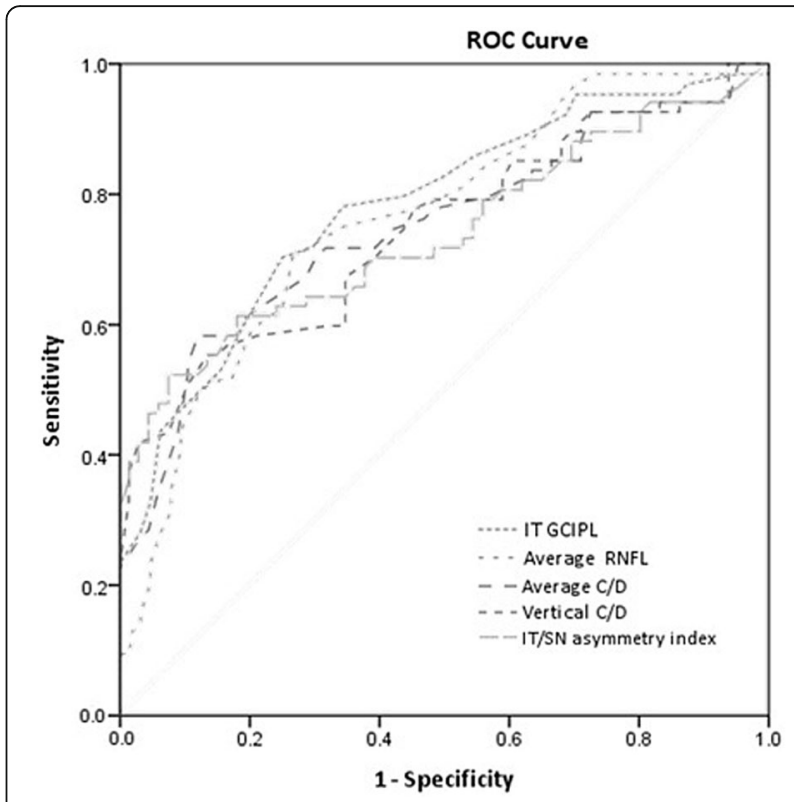

Fig. 2 AUROC values for PPG diagnosis. The inferotemporal (IT) GCIPL thickness showed the largest AUROC value (0.784), followed by average retinal nerve fiber layer (RNFL) thickness (0.767), average cup-to-disc ratio (C/D) (0.746), vertical C/D (0.742), and inferotemporal/superonasal (IT/SN) asymmetry index (0.734)

index. Based on the pAUROCs $\geq 90$ and $95 \%$ specificity, IT/SN asymmetry index had the largest pAUROC among all parameters (0.044 and 0.019 , respectively). Table 4 shows sensitivities at fixed specificities and cutoff values for all OCT parameters. At $90 \%$ specificity, IT/SN asymmetry index, average C/D and the cup volume had the highest sensitivity (all $52.2 \%$ ), followed by vertical C/D and IT-SN (both 50.7\%), and IT GCIPL (49.3\%). At $95 \%$ specificity, IT/SN asymmetry index had the highest diagnostic sensitivity (46.3\%), followed by vertical C/D (41.5\%) and IT/SN asymmetry ratio (40.3\%). Table 5 shows the $P$ values for pairwise comparison of AUROC values, pAUROC values, and sensitivity at fixed specificities between the best measure of each GCIPL asymmetry analysis and cpRNFL, GCIPL, and $\mathrm{ONH}$ parameters. The diagnostic performance was comparable between the best IT/SN asymmetry index and average RNFL thickness $(P=0.398)$, IT GCIPL thickness $(P=0.277)$, and average $\mathrm{C} / \mathrm{D}(P=0.618)$ based on AUROC values.

\section{Discussion}

In PPG eyes, we find the parameters with largest AUROCs were IT GCIPL thickness, average RNFL thickness, and average $\mathrm{C} / \mathrm{D}$. The diagnostic ability of the GCIPL parameters was similar to that of the RNFL and ONH parameters to differentiate from PPG from controls, as Sung et al. [19] and Kim et al. [20] have reported. In contrast, Begum and colleagues [21] show that the diagnostic ability of 
Table 4 Sensitivities at fixed specificities and cutoff values for cpRNFL thickness, GCIPL thickness, GCIPL asymmetry measurements, and $\mathrm{ONH}$ parameters

\begin{tabular}{|c|c|c|c|c|}
\hline Parametres & $\begin{array}{l}\text { Sensitivity at } 90 \% \text { specificity } \\
(\%)\end{array}$ & $\begin{array}{l}\text { Cutoff } \\
\text { value* }^{*}\end{array}$ & $\begin{array}{l}\text { Sensitivity at 95\% specificity } \\
(\%)\end{array}$ & $\begin{array}{l}\text { Cutoff } \\
\text { value** }^{*}\end{array}$ \\
\hline \multicolumn{5}{|l|}{ cpRNFL Thickness } \\
\hline Average & 41.7 & 84.5 & 23.3 & 80.5 \\
\hline Superior & 30.0 & 93.5 & 8.3 & 86.5 \\
\hline Nasal & 16.7 & 53.5 & 6.7 & 44.5 \\
\hline Inferior & 46.7 & 100.5 & 26.7 & 92.5 \\
\hline Temporal & 21.7 & 64.5 & 13.3 & 61.5 \\
\hline \multicolumn{5}{|l|}{ GCIPL Thickness } \\
\hline Average & 23.9 & 72.5 & 10.4 & 70.5 \\
\hline Minimum & 35.8 & 70.5 & 22.3 & 66.5 \\
\hline Superonasal & 11.9 & 72.5 & 9.0 & 70.5 \\
\hline Superior & 16.4 & 72.5 & 9.0 & 70.5 \\
\hline Superotemporal & 34.3 & 72.5 & 20.9 & 71.5 \\
\hline Inferotemporal & 49.3 & 73.5 & 34.3 & 71.5 \\
\hline Inferior & 20.9 & 68.5 & 19.4 & 66.5 \\
\hline Inferonasal & 13.4 & 71.5 & 6.0 & 66.5 \\
\hline \multicolumn{5}{|c|}{ GCIPL Absolute difference } \\
\hline I-S & 31.3 & 12.5 & 26.9 & 13.5 \\
\hline IT-ST & 38.8 & 4.5 & 32.8 & 6.5 \\
\hline $\mathrm{T}-\mathrm{N}$ & 29.9 & 14.5 & 23.9 & 15.5 \\
\hline IT-SN & 50.7 & 6.5 & 35.8 & 8.5 \\
\hline IT-IN & 29.9 & 7.5 & 26.9 & 8.5 \\
\hline ST-IN & 10.4 & 8.5 & 10.4 & 10.5 \\
\hline \multicolumn{5}{|c|}{ GCIPL Asymmetry Ratio } \\
\hline $1 / 5$ & 31.3 & 0.95 & 28.4 & 0.95 \\
\hline IT/ST & 35.8 & 0.96 & 28.4 & 0.95 \\
\hline $\mathrm{T} / \mathrm{N}$ & 46.3 & 0.94 & 35.8 & 0.92 \\
\hline IT/SN & 46.3 & 0.92 & 40.3 & 0.91 \\
\hline $\mathrm{IT} / \mathrm{IN}$ & 37.3 & 0.93 & 34.3 & 0.92 \\
\hline ST/IN & 23.9 & 0.93 & 16.4 & 0.91 \\
\hline \multicolumn{5}{|c|}{ GCIPL Asymmetry Index } \\
\hline $\log _{10}(1 / S)$ & 32.8 & 0.024 & 29.9 & 0.025 \\
\hline $\log _{10}(\mathrm{IT} / \mathrm{ST})$ & 38.8 & 0.028 & 34.3 & 0.034 \\
\hline $\log _{10}(T / N)$ & 37.3 & 0.037 & 25.4 & 0.045 \\
\hline $\log _{10}(\mathrm{IT} / \mathrm{SN})$ & 52.2 & 0.036 & 46.3 & 0.040 \\
\hline $\log _{10}(\mathrm{IT/IN})$ & 29.9 & 0.042 & 26.9 & 0.050 \\
\hline $\log _{10}(\mathrm{ST} / \mathrm{IN})$ & 11.9 & 0.049 & 10.4 & 0.059 \\
\hline \multicolumn{5}{|l|}{$\mathrm{ONH}$} \\
\hline Rim Area & 28.4 & 0.945 & 19.4 & 0.885 \\
\hline Disc Area & 13.4 & 2.54 & 3.0 & 2.84 \\
\hline Average C/D & 52.2 & 0.695 & 29.9 & 0.725 \\
\hline Vertical C/D & 50.7 & 0.665 & 41.8 & 0.685 \\
\hline Cup Volume & 52.2 & 0.336 & 29.2 & 0.467 \\
\hline
\end{tabular}

$A U R O C$, area under the receiver operating characteristic curves; GCIPL, ganglion cell-inner plexiform layer; $c p R N F L$, circumferential peripapillary retinal nerve fiber layer; $O N H$, optic nerve head; $C / D$, cup-to-disc ratio; I, inferior hemisphere; $S$, superior hemisphere; $I T$, inferotemporal; $S T$, superotemporal; $T$, temporal; $N$, nasal; $S N$, superonasal; $I N$, inferonasal

*Based on $90 \%$ specificity

**Based on $95 \%$ specificity 
GCIPL parameters was significantly lower than that of the RNFL and ONH parameters. The discrepancy might be explained by the fact that the axons of RGCs travelling within the RNFL show $100 \%$ convergence at the ONH, while the macula area only contain $50 \%$ of the total RGCs. OCT measures limited scan area of macular GCIPL, and any RGCs damage outside the elliptical annulus is less likely to be detected by the scan. Furthermore, the standard definition of glaucoma is based primarily on $\mathrm{ONH}$ and RNFL changes, rather than macular changes. The bias favoring the ONH and RNFL could underestimate the diagnostic ability of macular parameters [21], even though there is a growing evidence that early glaucomatous damage involves the macula. Using OCT, RGCs damage in the macula is as detectable as the RNFL damage in the classic arcuate regions [22], and the diagnostic ability of GCIPL parameters increased significantly if the RNFL defects are closer to the fovea [20]. The topographic characteristics (angular location and width) of RNFL defects may also affect the performance of OCT. Superotemporal and inferotemporal RNFL bundles tend to converge temporally with increasing myopia [23], so they are more likely to be detected by a macular GCIPL scan. Compared to emmetropic subjects in Begum's study [21], the PPG patients of Kim et al. [20] as well as in the present study had a mean refractive error of $-1.95 \pm 1.56 \mathrm{D}$ and -4.62 $\pm 3.40 \mathrm{D}$, respectively. GCIPL parameters have shown better diagnostic ability than either RNFL or ONH parameters in myopic PPG with a mean refractive error of -2.92 $\pm 3.07 \mathrm{D}$ [24]. GCIPL maps may have a better ability to detect early glaucomatous damage in the Asian population due to the higher prevalence of myopia compared to other populations.

Among GCIPL parameters, minimum GCIPL thickness had superior diagnostic performance than other parameters for detecting early glaucoma [19, 25]. However, our study showed no diagnostic advantages of minimum GCIPL thickness. This result might be explained by the inclusion of very early glaucomatous patients with minimal structural change. Also, OCT data was compared with the built-in normal database, abnormal thinned macular area defined on color-coded significance map might be misleading (i.e., in myopic eyes). Therefore, measurements of asymmetric GCIPL distribution might be an alternative method in diagnosing PPG, in addition to traditional cpRNFL thickness, GCIPL thickness and ONH parameters. Yamada et al. [26] and Hwang et al. [14] recently reported that inferior-to-superior ganglion cell asymmetry index had good diagnostic ability in PPG and early glaucoma. Similarly, diagnostic performance of the Spectralis macular hemifield asymmetry was comparable to sectoral cpRNFL thickness in early glaucoma $[11,12]$. However, GCIPL asymmetry could be underestimated in a simple comparison between the averaged values of two or three sectors of GCIPL thickness, such as the inferior hemisphere versus the superior hemisphere. Compared to previous "hemispheric-based" studies, "sector-based" asymmetry analysis was expressed as difference between each six parafoveal sectors in the present study. We found the IT/SN GCIPL asymmetry index best discriminated PPG from controls based on either pAUROC or diagnostic sensitivity at 90 and $95 \%$ specificity. To our knowledge, this is the first study to demonstrate this new diagnostic parameter for PPG. Topographically, GCIPL thinning occurred in the inferior and temporal portions of the macula in early glaucoma [27], and the IT GCIPL has shown to be the most sensitive sector [24]. In the macular area, the peak density of RGCs occurs at paracentral 3.7 degrees and is thicker in the superior portion than in the inferior portion of the macula [28]. Histological studies also show that the temporal and inferior sectors have fewer ganglion cells than the superior and nasal sectors [29]. The superiority of the IT/SN asymmetry index might be explained by the anatomic changes between inferotemporal sector and its counterpart (superonasal sector). Therefore, IT/SN asymmetry index appears to be a valuable parameter in diagnosing PPG.

These results show that the diagnostic ability of GCIPL asymmetry measurements was comparable to that of GCIPL thickness analysis for PPG, consistent with a previous asymmetry study [14]. Another study showed that GCIPL asymmetry had inferior diagnostic performance than that of the GCIPL thickness parameters [30], which might be due to the differences between absolute difference, ratio, and calculated index. The absolute difference directly reflects the amount of change, while the ratio indicates the relative amount of change considering the baseline level [14]. The asymmetry index further amplifies the relative amount of change by using a logarithmic calculation. It is less dependent on the severity of glaucoma than were thickness measurements. Particularly in the pre-perimetric stage, the amount of glaucomatous change is relatively small compared to the changes seen in other stages of glaucoma. Although the localized thinning of the ganglion cell layer might not affect the average thickness, it might substantially affect the asymmetry index. Therefore, the $\log _{10}$ asymmetry index appears to have an increased ability to detect glaucoma than simple difference and ratio, and could be a new indicator of early localized glaucomatous damage.

This study had several limitations. First, the sample size was relatively small. A future study of larger sample size should be conducted. Second, all of the study subjects were of Chinese ethnicity and therefore the results cannot necessarily be extrapolated to patients of other ethnicities. Third, the definition of PPG was for this study. The enrolled criteria could not ensure that all participants are PPG, only 
Table 5 values for pairwise comparison of AUROC values, PAUROC values, and sensitivities at fixed specificities between the best measure of each GCIPL asymmetry analysis and cpRNFL, GCIPL, and ONH parameters

\begin{tabular}{llllll}
\hline & AUROC & $\begin{array}{l}\text { pAUROC at 90\% } \\
\text { specificity }\end{array}$ & $\begin{array}{l}\text { PAUROC at 95\% } \\
\text { specificity }\end{array}$ & $\begin{array}{l}\text { Sensitivity at 90\% } \\
\text { specificity }\end{array}$ & $\begin{array}{l}\text { Sensitivity at 95\% } \\
\text { specificity }\end{array}$ \\
\hline IT-SN vs. IT GCIPL thickness & 0.277 & 0.151 & 0.340 & 0.474 & 0.767 \\
IT-SN vs. average cpRNFL thickness & 0.398 & 0.870 & 0.060 & 0.304 & 0.029 \\
IT-SN vs. average C/D & 0.618 & 0.441 & 0.484 & 0.667 & 0.225 \\
IT/IN vs. IT GCIPL thickness & 0.141 & 0.636 & 0.883 & 0.250 & 1.00 \\
IT/IN vs. average cpRNFL thickness & 0.247 & 0.268 & 0.141 & 0.553 & 0.059 \\
IT/IN vs. average C/D & 0.414 & 0.950 & 0.775 & 0.386 & 0.359 \\
Log (IT/SN) vs. IT GCIPL thickness & 0.395 & 0.226 & 0.290 & 0.322 & 0.083 \\
Log (IT/SN) vs. average cpRNFL & 0.705 & 0.017 & 0.011 & 0.007 & $<0.001$ \\
thickness & & & 0.025 & 0.203 & 0.008 \\
Log (IT/SN) vs. average C/D & 0.845 & 0.145 & &
\end{tabular}

$A U R O C$, area under the receiver operating characteristic curves; $P A U R O C$, partial area under the receiver operating characteristic curves; GCIPL, ganglion cell-inner plexiform layer; $c P R N F L$, circumferential peripapillary retinal nerve fiber layer; $C / D$, cup-to-disc ratio; $I T$, inferotemporal; $S N$, superonasal; $I N$, inferonasal

prospective follow-up could provide enough evidence for the diagnosis. No distinct evidence of progression could be observed to differentiate true PPG from glaucoma suspect because this is a cross-sectional observation study. Forth, we recruited PPG based on glaucomatous structural change and normal VF findings, which is defined as normal hemifield test that is symmetric around the horizontal meridian. This could exclude glaucomatous eyes with very early symmetric function change. This means that the diagnostic ability of asymmetry parameters may be underestimated compared with that of thickness parameters. On the contrary, we might have recruited a majority of eyes with RNFL defects more close to fovea or damage in the inferior macula, which may overestimate the diagnostic ability of IT/SN asymmetry index. Lastly, the influence of test-retest variability on the asymmetry analysis was not considered. The performance of the built-in software algorithm of HD-OCT may affect the results. Despite these limitations, the present results are relevant to discriminating between PPG and normal eyes in clinical practice. In addition, the asymmetry measurements don't require any special software and can be easily calculated, and the GCIPL asymmetry parameters are helpful for early detection of glaucoma.

\section{Conclusions}

In the present study, we used to spectral domain OCT to evaluate the diagnostic ability of the absolute difference, asymmetry ratio, and $\log _{10}$ asymmetry index between six parafoveal macular GCIPL thickness measurements in PPG patients, and compare the asymmetry analysis of GCIPL thickness with traditional cpRNFL, GCIPL, and ONH parameters in PPG patients. Our results showed the diagnostic ability of GCIPL asymmetry measurements was comparable to that of cPRNFL, GCIPL, and ONH analysis for eyes with PPG. The IT/SN asymmetry index performed best of all the asymmetry analyses and may serve as a new parameter for detecting early structural changes in PPG.

\section{Additional files}

Additional file 1: The original data of normal group. (XLSX $20 \mathrm{~kb})$

Additional file 2: The original data of PPG group. (XLSX 21 kb)

\begin{abstract}
Abbreviation
AL: axial length; AUROC: area under the receiver operating characteristic curves; C/D, VF: visual field; cup-to-disc ratio; CCT: central corneal thickness; $\mathrm{Cl}$ : confidence interval; cpRNFL: circumferential peripapillary retinal nerve fiber layer; D: diopters; GCIPL: ganglion cell-inner plexiform layer; I: inferior hemisphere; IN: inferonasal; IOP: intraocular pressure; IT: inferotemporal; MD: mean deviation; N: nasal; OCT: optical coherence tomography; ONH: optic nerve head; pAUROC: partial area under the receiver operating characteristic curves; PPG: Preperimetric glaucoma; PSD: pattern standard deviation; RGCs: retinal ganglion cells; S: superior hemisphere; SE: spherical equivalence; SN: superonasal; ST: superotemporal; T: temporal; VFI: visual field index
\end{abstract}

Funding

No.

Availability of data and materials

All datasets generated and/or analyzed during this study are included in this article and its supplementary information files.

\section{Authors' contributions}

MC, YC, YK, CJL participated in the design of the study. MC and HY analyzed and interpreted the data. MC, HY wrote the article. CJL critically revised the article. MC, YC, CH, YK collected the data. MC an HY were responsible for provision of materials and patients. $\mathrm{MC}$ and $\mathrm{HY}$ reviewed the literature. All authors read and approved the final manuscript.

Ethics approval and consent to participate

The study protocol was approved by the Institutional Review Board of Taipei general Hospital and was designed in accordance with the Declaration of Helsinki. Written informed consent was obtained from all subjects.

Competing interests

The authors declare that they have no competing interests. 


\section{Publisher's Note}

Springer Nature remains neutral with regard to jurisdictional claims in published maps and institutional affiliations.

\section{Author details}

'Department of Ophthalmology, Taipei Veterans General Hospital, 201, Section 2, Shih-Pai Road, Taipei 11217, Taiwan. ${ }^{2}$ School of Medicine, National Yang-Ming University, Taipei, Taiwan. ${ }^{3}$ Institute of Clinical Medicine, National Yang-Ming University, Taipei, Taiwan.

Received: 8 May 2018 Accepted: 26 December 2018 Published online: 08 January 2019

\section{References}

1. Harwerth RS, Carter-Dawson L, Shen F, Smith EL 3rd, Crawford ML. Ganglion cell losses underlying visual field defects from experimental glaucoma. Invest Ophthalmol Vis Sci. 1999;40(10):2242-50

2. Quigley HA, Addicks EM, Green WR. Optic nerve damage in human glaucoma. III. Quantitative correlation of nerve fiber loss and visual field defect in glaucoma ischemic neuropathy, papilledema, and toxic neuropathy. Arch Ophthalmol. 1982;100(1):135-46.

3. Quigley HA, Dunkelberger GR, Green WR. Retinal ganglion cell atrophy correlated with automated perimetry in human eyes with glaucoma. Am J Ophthalmol. 1989:107(5):453-64.

4. Harwerth RS, Carter-Dawson L, Smith EL 3rd, Barnes G, Holt WF, Crawford ML. Neural losses correlated with visual losses in clinical perimetry. Invest Ophthalmol Vis Sci. 2004;45(9):3152-60.

5. Tan O, Chopra V, Lu AT, Schuman JS, Ishikawa H, Wollstein G, Verma R, Huang D. Detection of macular ganglion cell loss in glaucoma by Fourierdomain optical coherence tomography. Ophthalmology. 2009;116(12):230514. https://doi.org/10.1016/j.ophtha.2009.05.025.

6. Kotera $Y$, Hangai M, Hirose F, Mori S, Yoshimura N. Three-dimensional imaging of macular inner structures in glaucoma by using spectral-domain optical coherence tomography. Invest Ophthalmol Vis Sci. 2011;52(3):141221. https://doi.org/10.1167/iovs.10-5572.

7. Lisboa R, Paranhos A Jr, Weinreb RN, Zangwill LM, Leite MT, Medeiros FA. Comparison of different spectral domain OCT scanning protocols for diagnosing preperimetric glaucoma. Invest Ophthalmol Vis Sci. 2013;54(5): 3417-25. https://doi.org/10.1167/iovs.13-11676.

8. Mwanza JC, Durbin MK, Budenz DL, Sayyad FE, Chang RT, Neelakantan A, Godfrey DG, Carter R, Crandall AS. Glaucoma diagnostic accuracy of ganglion cell-inner plexiform layer thickness: comparison with nerve fiber layer and optic nerve head. Ophthalmology. 2012;119(6):1151-8. https://doi. org/10.1016/j.ophtha.2011.12.014.

9. Hwang $\mathrm{YH}$, Jeong YC, Kim HK, Sohn YH. Macular ganglion cell analysis for early detection of glaucoma. Ophthalmology. 2014;121(8):1508-15. https:// doi.org/10.1016/j.ophtha.2014.02.019.

10. Asrani S, Rosdahl JA, Allingham RR. Novel software strategy for glaucoma diagnosis: asymmetry analysis of retinal thickness. Arch Ophthalmol. 2011;129(9):1205-11. https://doi.org/10.1001/ archophthalmol.2011.242.

11. Seo JH, Kim TW, Weinreb RN, Park KH, Kim SH, Kim DM. Detection of localized retinal nerve fiber layer defects with posterior pole asymmetry analysis of spectral domain optical coherence tomography. Invest Ophthalmol Vis Sci. 2012;53(8): 4347-53. https://doi.org/10.1167/iovs.12-9673.

12. Um TW, Sung KR, Wollstein G, Yun SC, Na JH, Schuman JS. Asymmetry in hemifield macular thickness as an early indicator of glaucomatous change. Invest Ophthalmol Vis Sci. 2012;53(3):1139-44. https://doi.org/10.1167/iovs.11-8373.

13. Sullivan-Mee M, Ruegg CC, Pensyl D, Halverson K, Qualls C. Diagnostic precision of retinal nerve fiber layer and macular thickness asymmetry parameters for identifying early primary open-angle glaucoma. Am J Ophthalmol. 2013;156(3):567-77. https://doi.org/10.1016/j.ajo.2013.04.037.

14. Hwang YH, Ahn SI, Ko SJ. Diagnostic ability of macular ganglion cell asymmetry for glaucoma. Clin Exp Ophthalmol. 2015;43(8):720-6. https://doi.org/10.1111/ceo. 12545

15. Kim YK, Yoo BW, Kim HC, Park KH. Automated detection of hemifield difference across horizontal raphe on ganglion cell-Inner plexiform layer thickness map. Ophthalmology. 2015;122(11):2252-60. https://doi.org/10.1016/j.ophtha.2015.07.013.

16. Sharifipour F, Morales E, Lee JW, Giaconi J, Afifi AA, Yu F, Caprioli J, Nouri-Mahdavi K. Vertical macular asymmetry measures derived from
SD-OCT for detection of early glaucoma. Invest Ophthalmol Vis Sci. 2017;58(10):4310-7. https://doi.org/10.1167/iovs.17-21961.

17. Budenz DL. Altas of visual fields. Philadelphia, PA: Lippinocott-Raven; 1997. p. 143-5.

18. DeLong ER, DeLong DM, Clarke-Pearson DL. Comparing the areas under two or more correlated receiver operating characteristic curves: a nonparametric approach. Biometrics. 1988;44(3):837-45.

19. Sung MS, Yoon JH, Park SW. Diagnostic validity of macular ganglion cell-inner plexiform layer thickness deviation map algorithm using cirrus HD-OCT in preperimetric and early glaucoma. J Glaucoma. 2014;23(8): 144-51. https://doi.org/10.1097/IJG.0000000000000028.

20. Kim MJ, Jeoung JW, Park KH, Choi YJ, Kim DM. Topographic profiles of retinal nerve fiber layer defects affect the diagnostic performance of macular scans in preperimetric glaucoma. Invest Ophthalmol Vis Sci. 2014;55(4):2079-87. https://doi.org/10.1167/iovs.13-13506.

21. Begum VU, Addepalli UK, Yadav RK, Shankar K, Senthil S, Garudadri CS, Rao HL Ganglion cell-inner plexiform layer thickness of high definition optical coherence tomography in perimetric and preperimetric glaucoma. Invest Ophthalmol Vis Sci. 2014;55(8):4768-75. https://doi.org/10.1167/iovs.14-14598.

22. Hood DC, Raza AS, de Moraes CG, Liebmann JM, Ritch R. Glaucomatous damage of the macula. Prog Retin Eye Res. 2013;32:1-21. https://doi.org/10. 1016/j.preteyeres.2012.08.003.

23. Leung CK, Yu M, Weinreb RN, Mak HK, Lai G, Ye C. LamDS. Retinal nerve fiber layer imaging with spectral-domain optical coherence tomography: interpreting the RNFL maps in healthy myopic eyes. Invest Ophthalmol Vis Sci. 2012;53(11):7194-200. https://doi.org/10.1167/iovs.12-9726.

24. Seol BR, Jeoung JW, Park KH. Glaucoma detection ability of macular ganglion cell-inner plexiform layer thickness in myopic preperimetric glaucoma. Invest Ophthalmol Vis Sci. 2015;56(13):8306-13. https://doi.org/ 10.1167/iovs.15-18141.

25. Mwanza JC, Budenz DL, Godfrey DG, Neelakantan A, Sayyad FE, Chang RT, Lee RK. Diagnostic performance of optical coherence tomography ganglion cell-inner plexiform layer thickness measurements in early glaucoma. Ophthalmology. 2014;121(4):849-54. https://doi.org/10.1016/j.ophtha.2013. 10.044 .

26. Yamada H, Hangai M, Nakano N, Takayama K, Kimura Y, Miyake M, Akagi T, Ikeda HO, Noma H, Yoshimura N. Asymmetry analysis of macular inner retinal layers for glaucoma diagnosis. Am J Ophthalmol. 2014;158(6):131829. https://doi.org/10.1016/j.ajo.2014.08.040.

27. Hood DC, Raza AS, de Moraes CG, Johnson CA, Liebmann JM, Ritch R. The nature of macular damage in glaucoma as revealed by averaging optical coherence tomography data. Transl Vis Sci Technol. 2012;1(1):3.

28. Watson $A B$. A formula for human retinal ganglion cell receptive field density as a function of visual field location. J Vis. 2014;14(7):1-17. https://doi.org/10. 1167/14.7.15.

29. Curcio CA, Allen KA. Topography of ganglion cells in human retina. J Comp Neurol. 1990:300(1):5-25

30. Park JW, Jung HH, Heo H, Park SW. Validity of the temporal-to-nasal macular ganglion cell-inner plexiform layer thickness ratio as a diagnostic parameter in early glaucoma. Acta Ophthalmol. 2015;93(5):e356-65. https://doi.org/10. 1111/aos.12666

\section{Ready to submit your research? Choose BMC and benefit from:}

- fast, convenient online submission

- thorough peer review by experienced researchers in your field

- rapid publication on acceptance

- support for research data, including large and complex data types

- gold Open Access which fosters wider collaboration and increased citations

- maximum visibility for your research: over $100 \mathrm{M}$ website views per year

At BMC, research is always in progress.

Learn more biomedcentral.com/submission 